\title{
Role of High-affinity Choline Transporter 1 in Colonic Hypermotility in a Rat Model of Irritable Bowel Syndrome
}

\author{
Meng-juan Lin ${ }^{1,2}$ and Bao-ping Yu ${ }^{1,2 *}$ \\ ${ }^{I}$ Department of Gastroenterology, Renmin Hospital of Wuhan University, Wuhan, Hubei, China; and ${ }^{2}$ Key Laboratory of Hubei Province for \\ Digestive System Diseases, Wuhan, Hubei, China
}

\section{Background/Aims}

Irritable bowel syndrome (IBS) is a common disease characterized by intestinal dysmotility, the mechanism of which remains elusive. We aim to determine whether the high-affinity choline transporter 1 (CHT1), a determinant of cholinergic signaling capacity, modulates intestinal motility associated with stress-induced IBS.

\section{Methods}

A rat IBS model was established using chronic water avoidance stress (WAS). Colonic pathological alterations were evaluated histologically and intestinal motility was assessed by intestinal transit time and fecal water content (FWC). Visceral sensitivity was determined by visceromotor response to colorectal distension. RT-PCR, western blotting, and immunostaining were performed to identify colonic CHT1 expression. Contractility of colonic muscle strips was measured using isometric transducers. enzyme-linked immunosorbent assay was used to measure acetylcholine (ACh). We examined the effects of MKC-231, a choline uptake enhancer, on colonic motility.

\section{Results}

After 10 days of WAS, intestinal transit time was decreased and fecal water content increased. Visceromotor response magnitude in WAS rats in response to colorectal distension was significantly enhanced. Protein and mRNA CHT1 levels in the colon were markedly elevated after WAS. The density of CHT1-positive intramuscular interstitial cells of Cajal and myenteric plexus neurons in WAS rats was higher than in controls. Ammonium pyrrolidine dithiocarbamate partly reversed CHT1 upregulation and alleviated colonic hypermotility in WAS rats. Pharmacological enhancement of CHT1 activity by MKC-231 enhanced colonic motility in control rats via upregulation of $\mathrm{CHT} 1$ and elevation of ACh production.

\section{Conclusion}

Upregulation of CHT1 in intramuscular interstitial cells of Cajal and myenteric plexus neurons is implicated in chronic stress-induced colonic hypermotility by modulation of ACh synthesis via nuclear factor-kappa B signaling.

(J Neurogastroenterol Motil 2018;24:643-655)

\section{Key Words}

Choline tranporter; Gastrointestinal motility; Interstitial cells of Cajal; Irritable bowel syndrome; Myenteric plexus

Received: March 3, 2018 Revised: July 13, 2018 Accepted: July 31, 2018

() This is an Open Access article distributed under the terms of the Creative Commons Attribution Non-Commercial License (http://creativecommons. org/licenses/by-nc/4.0) which permits unrestricted non-commercial use, distribution, and reproduction in any medium, provided the original work is properly cited.

*Correspondence: Bao-ping Yu, MD, PhD

Department of Gastroenterology, Renmin Hospital of Wuhan University, No.238 Jiefang Rd, Wuhan, Hubei 430060, China Tel: +86-27-68759391, Fax: +86-27-68759391, E-mail: yubp62@163.com 


\section{Introduction}

The cholinergic nervous system is widely distributed within the enteric nervous system (ENS). ${ }^{1}$ Cholinergic neurons, which affect the amplitude and frequency of slow waves in the interstitial cells of Cajal (ICCs), ${ }^{2}$ are the major pathway of excitatory transmission within the ENS. ${ }^{1}$ Accumulating evidence indicates that the cholinergic system plays an important role in the regulation of gastrointestinal (GI) motility and that dysfunction of this system can cause dysmotility. ${ }^{1}$

Irritable bowel syndrome (IBS), which is characterized by intestinal dysmotility and visceral hyperalgesia, is a common functional GI disorder that affects up to $29.2 \%$ of the general population according to the Rome III criteria. ${ }^{3}$ Currently, the pathological mechanism of IBS is poorly understood. Previous reports have indicated that dysfunction of the cholinergic system within the ENS, such as heightened cholinergic-mediated short circuit current responses, the persistence of choline acetyltransferase-positive neurons, enhanced mucosal acetylcholinesterase activity, and downregulation of the mucosal muscarinic 3 receptor, represents an important candidate mechanism for intestinal dysmotility in $\mathrm{IBS}^{4}$ Several lines of evidence suggest that the high-affinity choline transporter 1 (CHT1), which is the rate-limiting step in acetylcholine (ACh) synthesis, exerts a crucial role in the functioning of the cholinergic system. ${ }^{5,6} \mathrm{CHT} 1$, which is exclusively present in cholinergic nerve fibres, represents another biomarker of cholinergic nerves. ${ }^{7} \mathrm{CHT} 1$ is present on the presynaptic terminal of cholinergic neurons and can take up choline hydrolyzed from $\mathrm{ACh}$ at the synaptic cleft into the presynaptic nerve terminal. ${ }^{5,6}$ This choline uptake, which is $\mathrm{Na}^{+}$ and $\mathrm{Cl}^{-}$-dependent, is sensitive to hemicholinium-3. ${ }^{5,6} \mathrm{~A}$ previous study showed that ACh failed to be synthesized in CHT1 knockout mice. ${ }^{8}$ However, coluracetam (MKC-231), a choline uptake enhancer, was reported to improve cholinergic hypofunction by enhancing high-affinity choline uptake (HACU), subsequently facilitating ACh synthesis and release. ${ }^{9}$ To date, numerous studies have reported that altered expression and dysfunction of $\mathrm{CHT} 1$ plays a key role in the development of neurodegenerative diseases (especially Alzheimer's disease ${ }^{10}$ ), cardiovascular diseases (carotid atherosclerosis ${ }^{11}$ and tachycardia ${ }^{12}$ ), and tumours (such as pancreatic ductal adenocarcinoma $^{13}$ ). Our recent study reported for the first time that CHT1 might exert an important inhibitory effect in the visceral hyperalgesia induced by chronic pancreatitis. ${ }^{14}$

No reports regarding the involvement of CHT1 in the modulation of GI motility have been published to date. Herein, the present study aims to explore the alterations in the expression and distribution of $\mathrm{CHT} 1$ in the colon and to determine the role of $\mathrm{CHT} 1$ in intestinal dysmotility in a rat model of IBS induced by chronic stress.

\section{Materials and Methods}

\section{Animal Models}

Male Sprague-Dawley rats (weight 180-230 g) were purchased from Hunan SJA Laboratory Animal Co. Ltd. The animals were housed in a standardized environment $\left(22 \pm 2{ }^{\circ} \mathrm{C}\right.$ with a 12 -hour light/dark cycle and a relative humidity of 40-60\%), and had ad libitum access to food and water. All experiments were approved by the Institutional Animal Care and Use Committee of Wuhan University (No. 20170917) and conducted in accordance with the Declaration of the National Institutes of Health Guide for Care and Use of Laboratory Animals and the People's Republic of China animal welfare legislations in order to minimize the number of experimental animals and their suffering.

The rats were randomly divided into 4 groups: control group, water avoidance stress (WAS) group, MKC-231 group, and ammonium pyrrolidine dithiocarbamate (PDTC) group. The WAS procedure was performed to induce IBS as described previously with minor modifications. ${ }^{15}$ Briefly, rats were placed on a platform $(10 \times 8 \times 8 \mathrm{~cm}$; length $\times$ width $\times$ height $)$ in the center of a water-filled $\left(25^{\circ} \mathrm{C}\right) \operatorname{tank}(45 \times 25 \times 35 \mathrm{~cm}$; length $\times$ width $\times$ height) for 1 hour daily for 10 consecutive days. The water level in the tank was kept at $1 \mathrm{~cm}$ below the platform. Rats in the MKC231 group were the normal rats gavaged with MKC-231 (2 mg/ kg; MCE, Princeton, NJ, USA) for 10 days. The animals from the PDTC group were administered an intraperitoneal injection of PDTC (100 mg/kg; Beyotime, Shanghai, China), a selective nuclear factor-kappa B (NF- $\kappa \mathrm{B})$ antagonist, in saline 1 hour before WAS for 10 days. After 48 hours from the end of WAS, colonic motility and visceral sensitivity were determined. Afterwards, the rats were subjected to laparotomy and distal colon resection. Hematoxylin-eosin staining was performed for colon specimens.

\section{Intestinal Transit Time}

The animals were orally gavaged with activated carbon in double-distilled water after fasting for 24 hours. Close observation of the stool was then conducted. Intestinal transit time (ITT) was the duration from gavage to the time when the first black fecal pellet output. 


\section{Fecal Water Content}

Fecal water content (FWC) was used to estimate colonic motility as a validated index. The animals were placed in metabolic cages for 24 hours with free access to rodent chow and water. The stool was weighed $\left(\mathrm{m}_{0}\right)$ after collection, and the stool was then weighed again $\left(\mathrm{m}_{1}\right)$ after it was dried in the oven. $\mathrm{FWC}$ was calculated as $\left(\mathrm{m}_{0}\right.$ $\left.-\mathrm{m}_{1}\right) / \mathrm{m}_{0}$.

\section{Electromyogram Measurements}

To evaluate the visceral sensitivity, we recorded the electromyogram (EMG) signal of abdominal oblique musculature. For EMG measurements, animals were initially anesthetized with isoflurane inhalation, keeping a mild and stable anesthesia throughout the experiment. After anesthesia, the rat was fixed in a supine position. A pair of electrodes was implanted into the external oblique muscle of the rats. The electrodes were connected to a Bio Amp (AD instruments, Bella Vista, Australia) which was connected to a Power Lab (AD instruments) as an EMG acquisition system. Colorectal distension (CRD) was then conducted after 20 minutes of adaptation. Each recording progression consisted of a 5-minute predistention baseline activity measurements, a 20-second CRD-evoked response $(20,40,60$, and $80 \mathrm{mmHg})$, and a 3-minute postdistention activity measurement, followed by a 3-minute rest between $2 \mathrm{CRD}$ episodes. The EMG signals expressed by visceromotor response (VMR), the area under the curve in response to the CRD stimuli, were collected and analyzed using Lab Chart 7 software (AD instruments). The analytic period was 40 seconds (20 seconds during, and 20 seconds after each CRD). The net value for each CRD was calculated by subtracting the area under the curve of the baseline (40-second interval) before each CRD. ${ }^{16}$

\section{Western Blot}

Total proteins were extracted using RIPA lysis buffer (Beyotime) and subsequently subjected to centrifugation at $12000 \mathrm{rpm}$, $4^{\circ} \mathrm{C}$ for 30 minutes. Supernatants were then collected and protein concentration was determined using the BCA protein assay kit (Beyotime). Samples were mixed with $5 \times$ loading buffer and heated at $100^{\circ} \mathrm{C}$ for 5 minutes to denature the proteins. Thirty micrograms of total proteins were loaded on $10 \%$ SDS polyacrylamide gels and electrophoresed. The separated proteins were transferred to PVDF membranes (Millipore, Darmstadt, Germany), and the membranes were incubated in $5 \%$ skimmed milk at room temperature for 2 hours to block nonspecific binding. The blots were then incubated overnight at $4^{\circ} \mathrm{C}$ with the primary antibody against $\mathrm{CHT} 1$ (Santa
Cruz Biotechnology, Dallas, TX, USA) or $\beta$-actin (Beyotime). After washing 3 times with tris-buffered saline and Tween 20 (TBST) for 10 minutes, the corresponding secondary antibody conjugated to horseradish peroxidase (Boster) was applied for 1 hour at room temperature, followed by three washes of TBST for 10 minutes. Specific protein bands were visualized using the ECL kit (Thermo Fisher Scientific, Waltham, MA, USA) and an X-ray film (Kodak, Xiamen, China). The optical density of the bands was analyzed using Band Scan 5.0 software (Alpha Innotech Corp, San Leandro, CA, USA).

\section{Quantitative Real-time Polymerase Chain Reaction}

Total RNA was extracted from the colon that was frozen in liquid nitrogen with TRIzol reagent (Invitrogen, Carlsbad, CA, USA), following the manufacturer's instructions. Next, $1.5 \mu \mathrm{g}$ of total RNA from each sample was used for cDNA synthesis in a total volume of $20 \mu \mathrm{L}$, with oligo (dT)18 and Hiscript Reverse Transcriptase (Vazyme Biotech Co, Ltd, Nanjing, China) included in the reverse transcription system. Quantitative real-time polymerase chain reaction was performed in $20 \mu \mathrm{L}$ wells with SYBR Green PCR master mix (Vazyme Biotech Co, Ltd) on the viiA 7 real-time PCR system (Applied Biosystems Inc, Carlsbad, CA, USA). After incubation at $95^{\circ} \mathrm{C}$ for 10 minutes as the initiation of thermal cycling, 40 cycles of $95^{\circ} \mathrm{C}$ for 30 seconds and $60^{\circ} \mathrm{C}$ for 30 seconds were performed. Each reaction was performed in triplicate. Glyceraldehyde-3-phosphate dehydrogenase (GAPDH) was used as a loading control to normalize each sample. CHT1 is encoded by Slc5a7 gene. The primers for Slc5a7 amplification directly target the transcript variant $\mathrm{X} 3$, whose accession number is XM 006244253.3. The PCR primers were as follows: CHT1 forward: 5‘-GACTGTGTATGGGCTCTGGT-3', reverse: 5'-TGGCTCTCCTCCGGTAATTC-3'; GAPDH forward: 5'ACAGCAACAGGGTGGTGGAC-3', reverse: 5'-TTTGAGGGTGCAGCGAACTT-3'. Specificity of the PCR products was monitored by melting curve analysis. The relative level of CHT1 mRNA was quantified by the $2^{-\Delta \Delta \mathrm{Ct}}$ method.

\section{Immunofluorescence}

Immunofluorescence staining for CHT1 was performed both on whole-mount flat preparations of the myenteric plexus (MP) and longitudinal sections of the colon. Immunofluorescence staining on whole-mount flat preparations was performed as follows. Colonic specimens were fixed in $4 \%$ paraformaldehyde solution at $4^{\circ} \mathrm{C}$ for 6-8 hours. Subsequently, the mucosa was removed with sharp forceps under a stereoscopic microscope. The circular muscle 
was then stripped off carefully at certain intervals. Whole-mount preparations were then blocked with $10 \%$ goat serum containing $0.3 \%$ Triton $\mathrm{X}-100$ for 1 hour at room temperature. Next, the samples were incubated overnight at $4^{\circ} \mathrm{C}$ with mouse anti-rat polyclonal CHT1 antibody (Santa Cruz Biotechnology). After washing in phosphate buffered saline with Tween 20 (PBST), the pinned tissues were incubated overnight at $4^{\circ} \mathrm{C}$ with rabbit anti-rat polyclonal transmembrane member 16A (TMEM16A) antibody (Santa Cruz Biotechnology). After washing thrice with PBST for 5 minutes, the tissues were incubated with FITC-conjugated rabbit anti-mouse secondary antibody (Boster, Wuhan, China) and CY3-conjugated goat anti-rabbit secondary antibody (Boster) for 1 hour at room temperature. The stained samples were imaged using Olympus BX53 microscope (Olympus, Tokyo, Japan) after they were mounted on a slide with a coverslip, and sealed with glycerol. Immunofluorescence of colonic longitudinal sections was conducted in a similar manner with the above procedure. The results were analyzed using Image Pro Plus software version 6.0 (Media Cybernetics, Silver Spring, MD, USA). The CHT1-immunoreactive (IR) neurons in the MP were quantified as a relative percentage considering the total number of TMEM16A-IR neurons.

\section{Contractility of Colonic Muscle Strips}

Full-thickness strips of distal colon (measuring $3 \times 10 \mathrm{~mm}$ ) were mounted vertically in a $10 \mathrm{~mL}$ organ bath filled with Tyrode's solution (in mM: $\mathrm{NaCl} 147.0, \mathrm{KCl} 4.0, \mathrm{CaCl}_{2}$ 2.0, $\mathrm{MgCl}_{2}$ 1.05, $\mathrm{Na}_{2} \mathrm{HPO}_{4}$ 1.81, $\mathrm{NaH}_{2} \mathrm{PO}_{4}$ 0.42, and glucose 5.5, with $\mathrm{pH}$ adjusted to $7.35-7.45$ with $\mathrm{NaOH}$ ) maintained at $37^{\circ} \mathrm{C}$ and constantly bubbled with $\mathrm{O}_{2}$. The strips were placed under an initial resting tension equivalent to a $1.0 \mathrm{~g}$ load and allowed to equilibrate for 30 minutes, with solution changes every 20 minutes. Isometric contractions were measured using a force displacement transducer. The contraction curves were recorded and measured by RM6240 multichannel physiological signal acquisition and processing system (Chengdu Instrument Factory, Chengdu, China). The average magnitude and frequency were calculated to evaluate the spontaneous contraction of strips.

\section{Enzyme-linked Immunosorbent Assay}

Enzyme-linked immunosorbent assay (ELISA) was applied to detect the ACh release from the colon. For detecting ACh production, fresh colonic tissues were subjected to homogenization on ice after dilution, and subsequent centrifugation at 2500-3500 rpm for 10 minutes. Supernatants were then collected and ACh content was determined using the ACh ELISA Kit (Jiancheng, Nanjing,
China) according to the manufacturer's instructions. Each sample was assayed in duplicate. The spectrophotometry of panels were read at $550 \mathrm{~nm}$ and calculated according to the standard curve.

\section{Statistical Methods}

Statistical analyses were performed using SPSS version 21.0 (IBM Corp, Armonk, NY, USA). Continuous variables were presented as the mean \pm standard deviation and compared using $t$ tests and analysis of variance (ANOVA). Differences among the groups were analyzed using 2-way repeated-measures ANOVA with distention pressure as the repeated measure. The Student-NewmanKeuls post hoc test was used where appropriate. In all analyses, " $n$ " refers to the numbers of rats examined. Differences were considered statistically significant at a $P$-value $<0.05$.

\section{Results}

\section{Evaluation of the Animal Model}

No animals died during the experiment. Haematoxylin-eosin staining revealed that colon specimens of the control and WAS groups were intact, without congestion and obvious infiltration of inflammatory cells (Fig. 1A). However, the ITT of WAS rats was significantly shorter than that of controls $(7.90 \pm 0.24$ hours vs $10.10 \pm 0.32$ hours, $P<0.05 ; \mathrm{n}=5$ in each group) (Fig. 1B) Furthermore, FWC in WAS-exposed rats was enhanced compared to that in control rats $(70.69 \pm 6.61 \%$ vs $47.89 \pm 4.75 \%, P<$ $0.05 ; \mathrm{n}=5$ in each group) (Fig. $1 \mathrm{C}$ ). In addition, we detected that the VMR amplitude in the WAS group was markedly higher than that in the control group at the pressures of 20, 40, 60, and 80 $\mathrm{mmHg}(20 \mathrm{mmHg}$ : control $76.9 \pm 6.8 \mu \mathrm{V} \cdot \mathrm{sec}$ vs WAS $110.6 \pm$ $8.4 \mu \mathrm{V} \cdot \mathrm{sec}, P<0.05 ; 40 \mathrm{mmHg}$ : control $117.7 \pm 8.9 \mu \mathrm{V} \cdot \mathrm{sec}$ vs WAS $173.1 \pm 9.8 \mu \mathrm{V} \cdot \mathrm{sec}, P<0.05 ; 60 \mathrm{mmHg}$ : control $169.4 \pm$ $9.0 \mu \mathrm{V} \cdot \mathrm{sec}$ vs WAS $225.7 \pm 10.6 \mu \mathrm{V} \cdot \mathrm{sec}, P<0.05 ; 80 \mathrm{mmHg}$ : control $204.1 \pm 9.9 \mu \mathrm{V} \cdot \mathrm{sec}$ vs WAS $271.5 \pm 10.1 \mu \mathrm{V} \cdot \mathrm{sec}, P<$ $0.05 ; \mathrm{n}=5$ in each group) (Fig. 1D).

These data suggested that an animal model of chronic stressinduced colonic hypermotility, exhibiting the hallmark of IBS, was successfully established after 10 days of WAS exposure.

\section{Enhanced High-affinity Choline Transporter 1 Expression in the Colon of Water Avoidance Stress Rats}

Western blot analysis revealed that $\mathrm{CHT} 1$ protein levels in the colon were considerably increased after 10 days of WAS (Fig. 2A). The CHT1 and $\beta$-actin level ratios in the control and WAS groups 

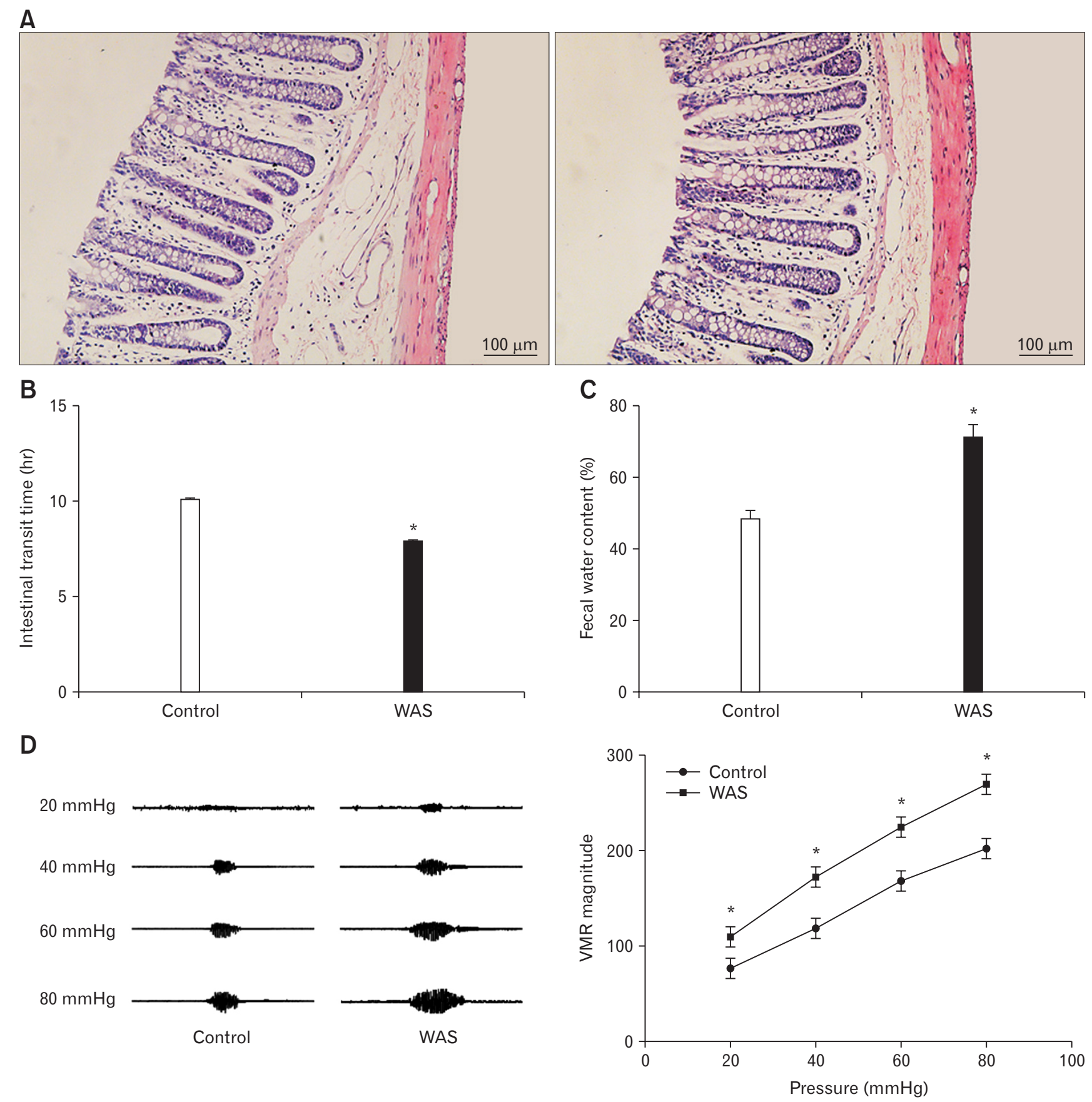

Figure 1. Evaluation of the animal model. (A) Hematoxylin-eosin staining $(\times 200)$ showing that colon specimens of the control (left) and WAS (right) groups are intact without congestion and obvious infiltration of inflammatory cells. (B) The intestinal transit time of water avoidance stress (WAS) rats is shorter than that of control rats. (C) The fecal water content in the WAS group is increased compared to that in the control group. (D) Visceromotor response (VMR) cure (left) and summary data (right) for VMR responses to CRD at pressures of 20, 40, 60, and 80 $\mathrm{mmHg}$ in control and WAS rats. The VMR amplitude of WAS rats is significantly higher than that of control rats. ${ }^{*} P<0.05$ compared to controls.

were $0.42 \pm 0.02$ and $0.72 \pm 0.04(P<0.05 ; \mathrm{n}=5$ in each group), respectively. Quantitative RT-PCR analysis further confirmed upregulation of CHT1 in the colon under chronic stress at the transcriptional level (Fig. 2B). The mean $2^{-\Delta \mathrm{Ct}}$ values of CHT1 mRNA levels in the control and WAS groups were $1.00 \pm 0.00$ and $1.80 \pm 0.05(P<0.05 ; \mathrm{n}=5$ in each group $)$, respectively. In addi- 
A

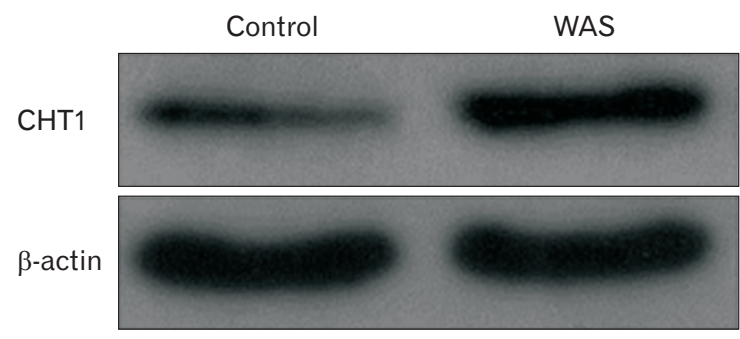

\section{B}

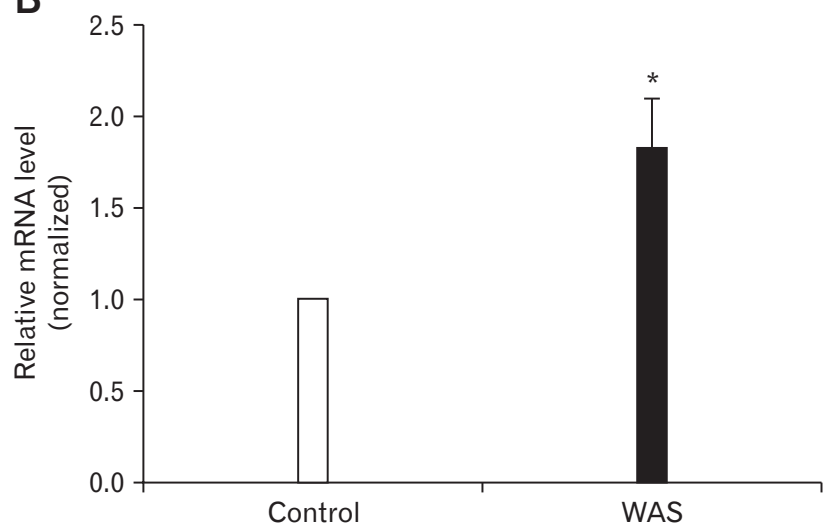

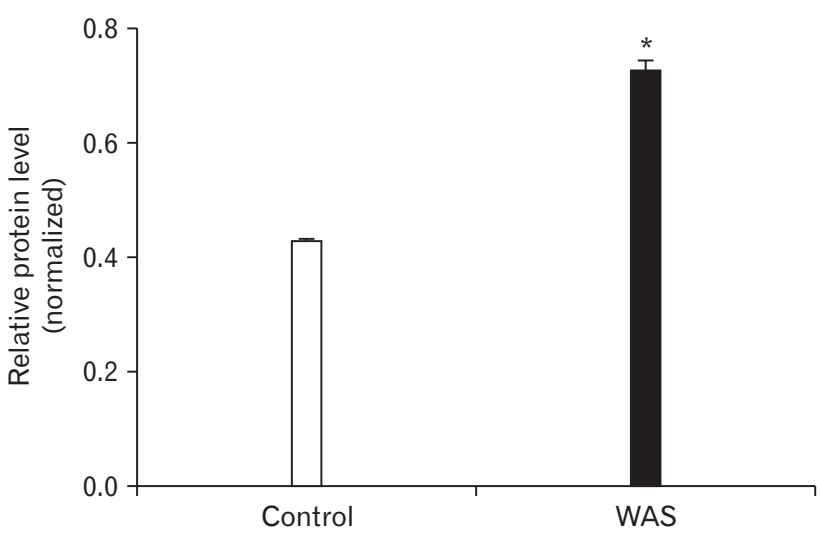

Figure 2. Protein and mRNA levels of high-affinity choline transporter 1 (CHT1) in the colon. (A) Western blot analysis revealing that CHT1 protein expression in water avoidance stress (WAS) rats is increased compared to that in control rats. (B) Quantitative real-time polymerase chain reaction analysis showing that the $\mathrm{CHT} 1 \mathrm{mRNA}$ levels of WAS rats are higher than those of control rats. ${ }^{*}<0.05$ compared to controls.

tion, we detected that TMEM16A protein/mRNA level in the colon was also markedly upregulated after 10 days of WAS treatment, consistent with the increasing trend of CHT1 (data not shown).

Our findings indicate that CHT1 expression in the colon was upregulated after 10 consecutive days of WAS exposure.

\section{Immunofluorescence Staining of High-affinity Choline Transporter 1 in the Colon}

Immunofluorescence staining of longitudinal colonic sections showed that CHT1 was robustly expressed in the colon, both in the mucosal and muscular layers (Fig. 3A). Moreover, we clearly observed two distinct populations of $\mathrm{CHT} 1$-positive cells in the muscular layer. One population was mainly fusiform in shape and found within the circular muscle, parallel to smooth muscle cells, exhibiting the morphological characteristics of ICC. The other population was predominantly round or oval in shape and located between the circular and longitudinal muscle, showing the hallmarks of MP neurons. Further double labelling staining of $\mathrm{CHT} 1$ with
TMEM16A revealed that both populations of $\mathrm{CHT} 1$-positive cells exhibited immunoreactivity to TMEM16A. Previous studies have suggested that TMEM16A is exclusively expressed in ICC of the GI muscle layer and exhibits more selective labeling of ICC than Kit antibodies. ${ }^{17}$ However, we demonstrated that TMEM16A immunoreactivity was exclusively present in the enteric neurons of the colonic MP (data not shown). In turn, our data indicated that CHT1 was exclusively expressed in the ICCs and enteric neurons of the MP in the colonic muscular layer. Further immunofluorescence analysis revealed an increased density of CHT1 labelling in the intramuscular ICC (ICC-IM) $(9.35 \pm 3.16$ vs $12.90 \pm 3.89$, $P<0.05 ; \mathrm{n}=4$ in each group) and MP neurons (6.42 \pm 2.33 vs $12.73 \pm 2.61, P<0.05 ; \mathrm{n}=4$ in each group) after 10 days of WAS treatment.

Next, we examined the distribution of CHT1-positive cells in the MP by stripping the circular muscle off whole-mount colonic specimens (Fig. 3B). The neuronal network-like structure and ganglia were clearly observed on the colonic MP whole-mounts 


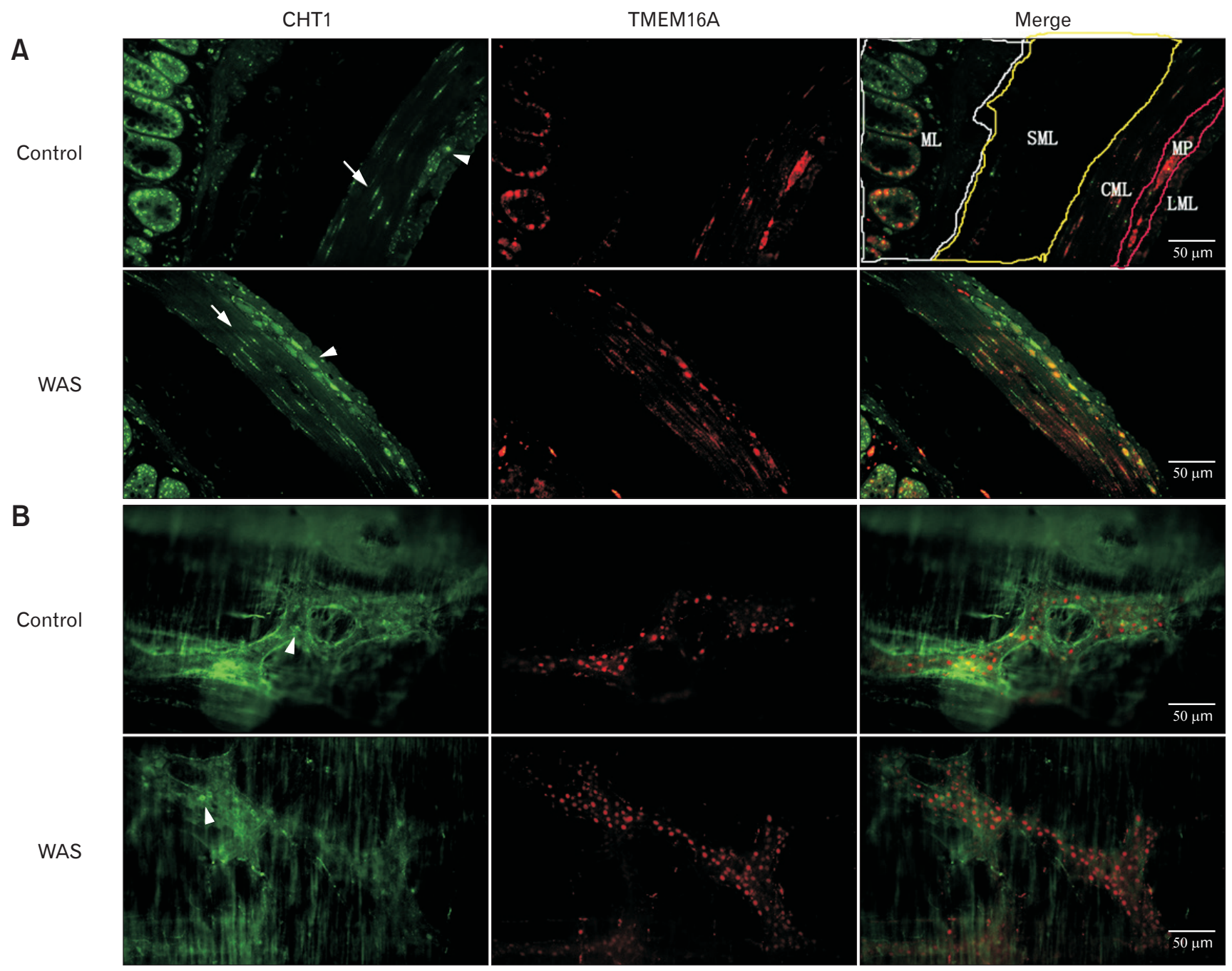

Figure 3. Immunostaining of high-affinity choline transporter 1 (CHT1) in the colon. (A) Double-immunostaining for CHT1 and transmembrane member 16A (TMEM16A) in longitudinal colonic sections from control and water avoidance stress (WAS) rats $(\times 400)$ revealing that CHT1 in the circular muscle of both naive and WAS rats is exclusively expressed in TMEM16A-immunoreactive (IR) cells and the number of CHT1-IR cells in the muscular layer of WAS rats is significantly increased compared to that of control rats. (B) Double-immunofluorescence on whole-mount preparations of colonic myenteric plexus $(\mathrm{MP})(\times 400)$ illustrating that TMEM16A is robustly expressed in colonic MP and that all the CHT1-IR cells are co-labelled with TMEM16A. The percentage of CHT1-IR cells in the MP is substantially elevated after 10 days of WAS. Different layers were marked out. White arrows indicate CHT1-positive cells that are mainly fusiform in shape and found within circular muscle, exhibiting the morphological characteristics of interstitial cells of Cajal. White arrowheads indicate CHT1-positive cells that are predominantly round in shape and located between circular and longitudinal muscles, showing the hallmark of MP neurons. ML, mucosal layer; SML, submucosal layer; CML, circular muscle layer; LML, longitudinal muscle layer.

after identifying neurons with TMEM16A. We observed that all the CHT1-positive cells were TMEM16A-IR. The CHT1IR cells in the MP were predominantly round or oval in shape. Furthermore, the percentage of CHT1-positive cells in the total TMEM16A-IR cells in the MP was substantially elevated after 10 days of WAS $(42.21 \pm 6.62 \%$ vs $65.63 \pm 7.25 \%, P<0.05 ; \mathrm{n}=$ 4 in each group).
These results indicate that the enhanced CHT1 expression observed in the colonic muscular layer of WAS rats was restricted to the ICC-IM and MP neurons.

\section{Effect of MKC-231 on Colonic Motility In Vivo}

MKC-231, a choline uptake enhancer, was administered to explore the role of CHT1 in intestinal motility. Western blot analysis 
revealed that $\mathrm{CHT} 1$ protein levels in the colon of control rats were considerably elevated after 10 consecutive days of MKC-231 treatment, with ratios of CHT1 to $\beta$-actin in the control and MKC-231 groups of $0.42 \pm 0.03$ and $0.68 \pm 0.04(P<0.05 ; \mathrm{n}=5$ in each group), respectively (Fig. 4A). Immunofluorescence further revealed that the density of $\mathrm{CHT} 1$ labelling in the colonic muscular layer was markedly increased after treatment with MKC-231 (Fig. 4B).

In vivo tests of GI motor function confirmed that the ITT of MKC-231-treated rats was significantly shorter than that of control rats $(8.40 \pm 0.22$ hours vs $10.10 \pm 0.32$ hours, $P<0.05$; $\mathrm{n}=5$ in each group) (Fig. 4C). In addition, FWC of the MKC-231 group was enhanced compared to the control group ( $64.17 \pm 5.87 \%$ vs $47.89 \pm 4.75 \%, P<0.05 ; \mathrm{n}=5$ in each group) (Fig. 4D).

These findings indicate that MKC-231 enhanced the colonic motility of healthy rats by upregulation of MKC-231 protein expression.

\section{Influence of MKC-231 on Spontaneous Contraction of Colonic Strips and Acetylcholine Production}

In vitro study of colonic strips recorded spontaneous contractility of the colon (Fig. 5A). Upon statistical analysis, we found that the amplitude $(1.42 \pm 0.15 \mathrm{~g}$ in control group vs $1.87 \pm 0.20 \mathrm{~g}$ in WAS group, $P<0.05 ; \mathrm{n}=5$ in each group) and frequency (2.41 \pm 0.13 minute $^{-1}$ in control group vs $3.52 \pm 0.15$ minute $^{-1}$ in WAS group, $P<0.05 ; \mathrm{n}=5$ in each group) of spontaneous contractions in WAS-exposed rats were significantly elevated compared to those in control rats, suggesting that colonic hypermotility was induced after 10 days of WAS (Fig. 5B and 5C). Furthermore, MKC-231 could markedly enhance the amplitude $(1.42 \pm 0.15 \mathrm{~g}$ in control group vs $1.75 \pm 0.24 \mathrm{~g}$ in MKC-231 group, $P<0.05 ; \mathrm{n}=5$ in each group) of spontaneous contractions in control rats. However, the frequency $\left(2.41 \pm 0.13\right.$ minute $^{-1}$ in control group vs 2.16 \pm 0.13 minute $^{-1}$ in MKC-231 group, $P>0.05 ; \mathrm{n}=5$ in each group) of spontaneous contractions in MKC-231-treated rats was almost equal to that in control rats.

Using ELISA, we detected that the ACh content in the colon of WAS-exposed rats was considerably increased ( $2.67 \pm 0.23 \mu \mathrm{g}$ ) $\mathrm{mg}$ in the control group vs $3.42 \pm 0.27 \mu \mathrm{g} / \mathrm{mg}$ in the WAS group, $P<0.05 ; \mathrm{n}=4$ in each group) (Fig. 5D). Notably, the ACh content in control rats was elevated after treatment with MKC-231 (2.67 $\pm 0.23 \mu \mathrm{g} / \mathrm{mg}$ in the control group vs $3.07 \pm 0.21 \mu \mathrm{g} / \mathrm{mg}$ in the MKC-231 group, $P<0.05 ; \mathrm{n}=4$ in each group).

These results suggest that MKC-231 elevated spontaneous contractions of colonic strips by enhancing ACh production.

\section{Effect of Ammonium Pyrrolidine Dithiocarbamate on High-affinity Choline Transporter 1 Upregulation and Colonic Hypermotility in Water Avoidance Stress-exposed Rats}

PDTC, a selective NF- $\kappa B$ antagonist, was administered to explore the mechanism of $\mathrm{CHT} 1$ upregulation in WAS-exposed rats. Western blot analysis revealed that the obtained CHT1 and $\beta$-actin level ratios in the WAS and PDTC groups were $0.72 \pm 0.06$ and $0.49 \pm 0.05,(P<0.05 ; \mathrm{n}=5$ in each group), respectively (Fig. $6 \mathrm{~A})$, indicating that $\mathrm{CHT} 1$ upregulation in the WAS-exposed group was largely abolished by PDTC.

Further in vivo study of GI motor function revealed that the ITT in the PDTC group was significantly longer than that in the WAS group $(9.10 \pm 0.29$ hours vs $7.90 \pm 0.24$ hours, $P<0.05$; $\mathrm{n}=5$ in each group) (Fig. 6B). Moreover, the FWC of PDTCtreated rats also displayed a downward trend compared to that in the WAS group $(59.11 \pm 5.46 \%$ vs $70.69 \pm 6.61 \%, P<0.05$; n $=5$ in each group) (Fig. $6 \mathrm{C}$ ).

These results indicate that inhibition of NF- $\mathrm{BB}$ signaling relieved stress-induced colonic hypermotility partly via the CHT1 downregulation.

\section{Discussion}

Currently, the detailed mechanism of IBS remains elusive. The known mechanisms implicated in its pathophysiology include disrupted gut-brain axis, ${ }^{18}$ visceral hypersensitivity, ${ }^{19}$ altered gut microbiota, ${ }^{20}$ epithelial barrier dysfunction, ${ }^{21}$ and immune activation. ${ }^{22}$ Animal models of IBS are pivotal in clarifying its pathogenesis. To date, repeated WAS has been utilized to establish an animal model of stress-induced IBS with visceral hypersensitivity, motility impairment, anxiety, and colonic immune activation, which can persist for the following 20 days. ${ }^{23}$ The optimal WAS model for studies on IBS is a brain-gut interaction model that mimics some clinical and pathophysiological characteristics of IBS-diarrhea. ${ }^{24}$ In the current study, elevated intestinal motor function and visceral hypersensitivity in response to $\mathrm{CRD}$ were observed after 10 days of WAS exposure, indicating that an animal model of stress-induced IBS was successfully established.

The cholinergic system is widely distributed in the ENS and is involved in the regulation of GI motility, ${ }^{1}$ secretion, sensory signaling, and vascular reflexes. ${ }^{25}$ The cholinergic along with tachykinergic neurons are the 2 major pathways of excitatory transmission within the ENS. ${ }^{1}$ Accumulating evidence has suggested that dysfunction of the cholinergic system in the ENS may be relevant 


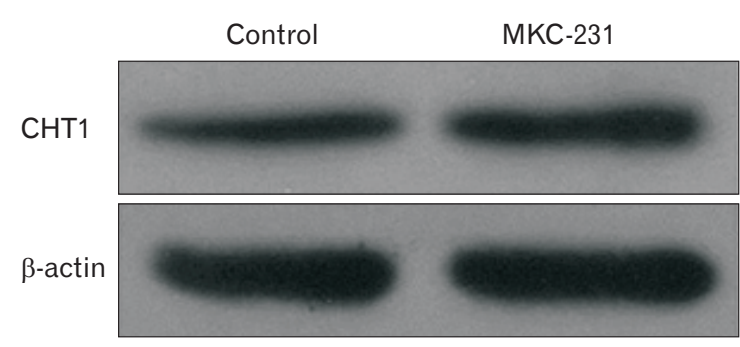

B

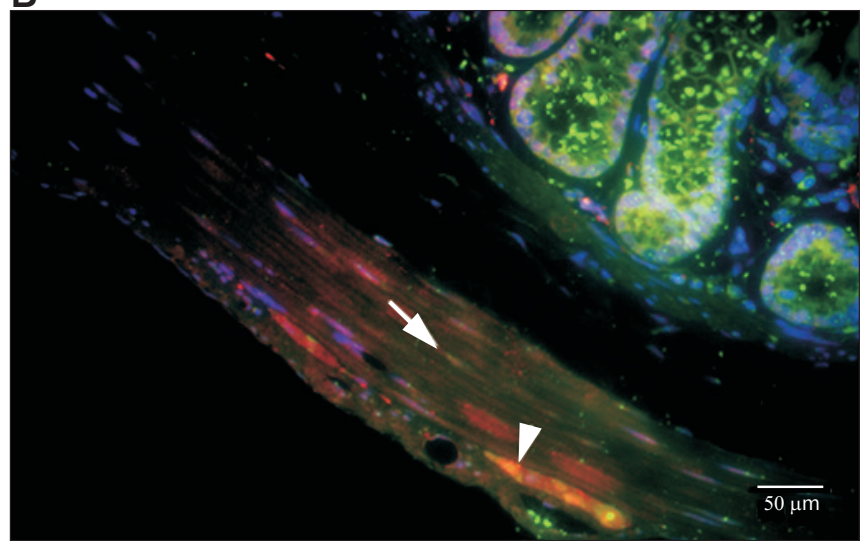

C

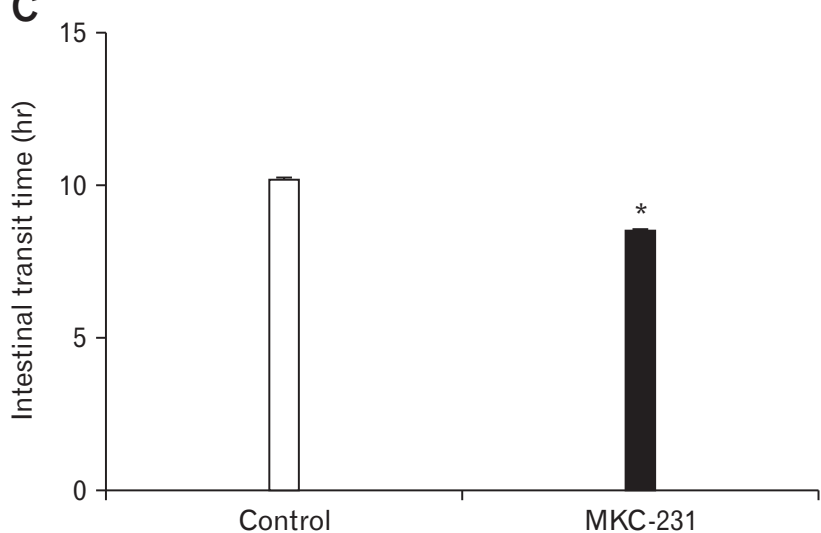

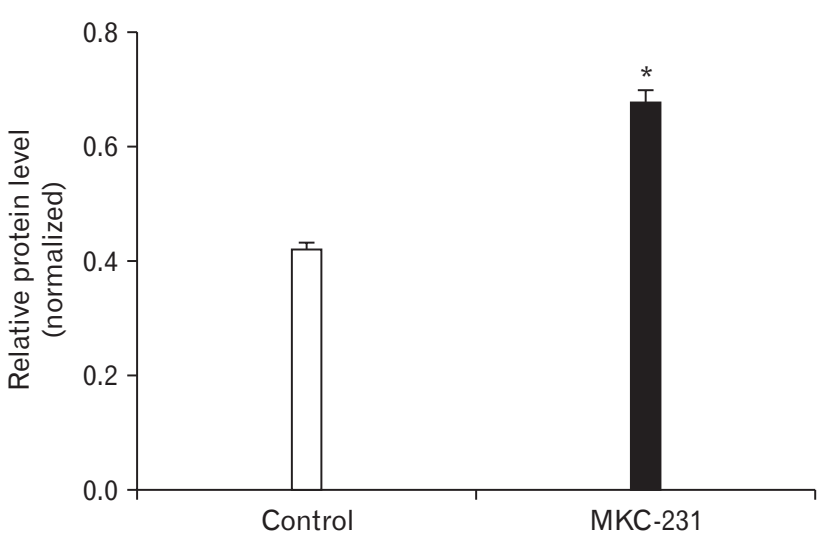

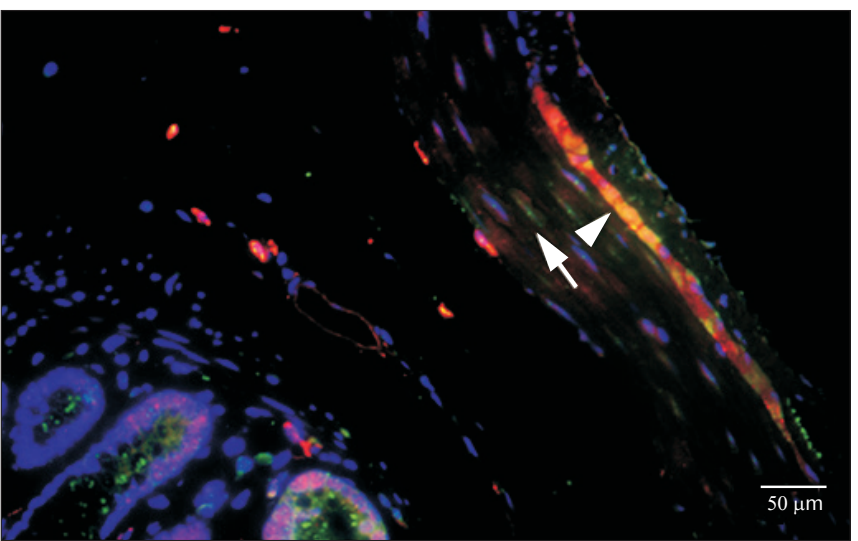

MKC-231

D

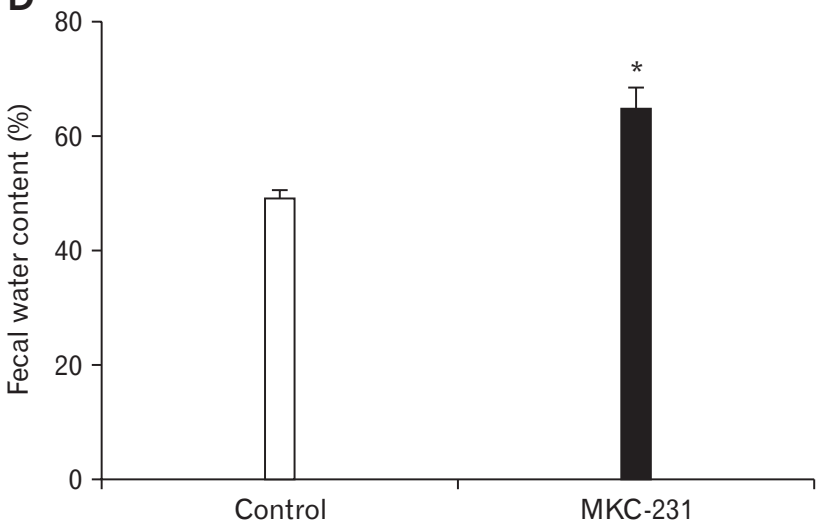

Figure 4. Influence of MKC-231, a choline uptake enhancer, on high-affinity choline transporter 1 (CHT1) expression and intestinal motility in vivo. (A) Immunoblots (left) and histogram (right) for CHT1 in the colon from control and MKC-231-treated rats showing that the ratios of CHT1 to $\beta$-actin expression in control rats are significantly increased after 10 consecutive days of MKC-231 treatment. (B) Merged pictures of immunostaining for CHT1, transmembrane member 16A (TMEM16A), and DAPI (4',6-diamidino-2-phenylindole) in longitudinal colonic sections from control (left) and MKC-231 rats (right) $(\times 400)$ revealing that the density of CHT1 labelling in the colonic muscular layer is considerably elevated after treatment with MKC-231. White arrows indicate CHT1-positive intramuscular interstitial cells of Cajal. White arrowheads indicate CHT1-immunoreactive (IR) neurons in the myenteric plexus. (C) The intestinal transit time of MKC-231-rats is reduced compared to that of control rats. (D) The fecal water content of the MKC-231 group is considerably increased compared with that of the control group. ${ }^{*} P<0.05$ compared to controls. 
A
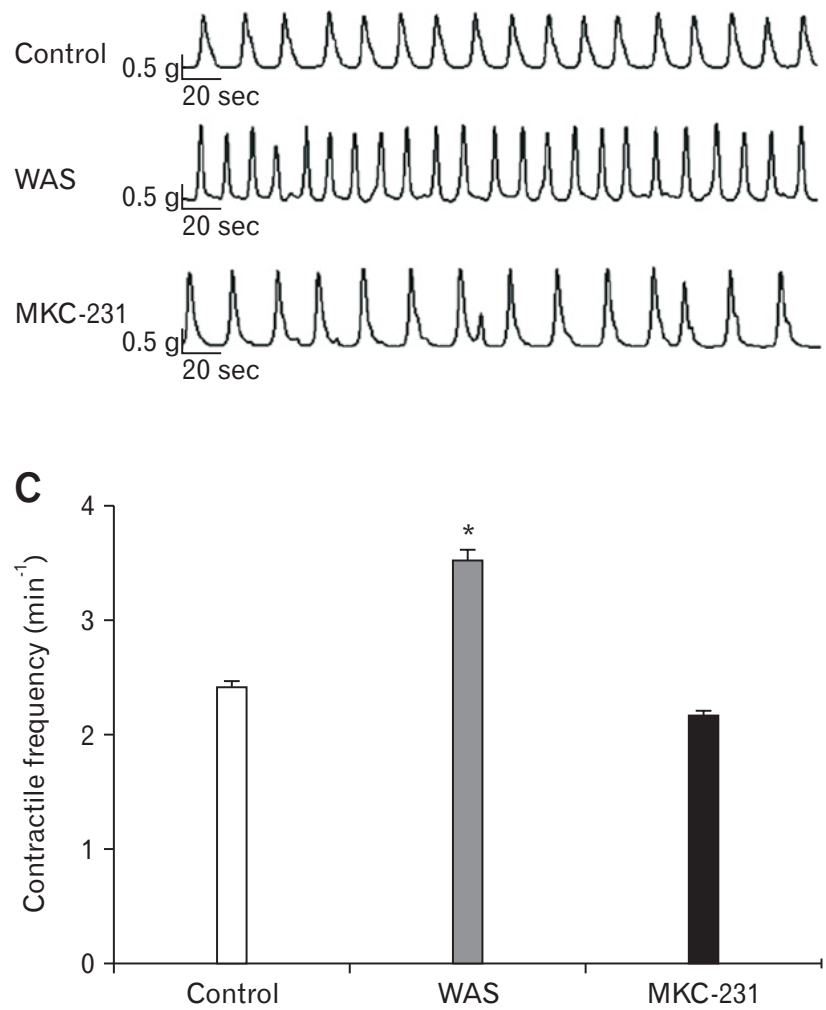
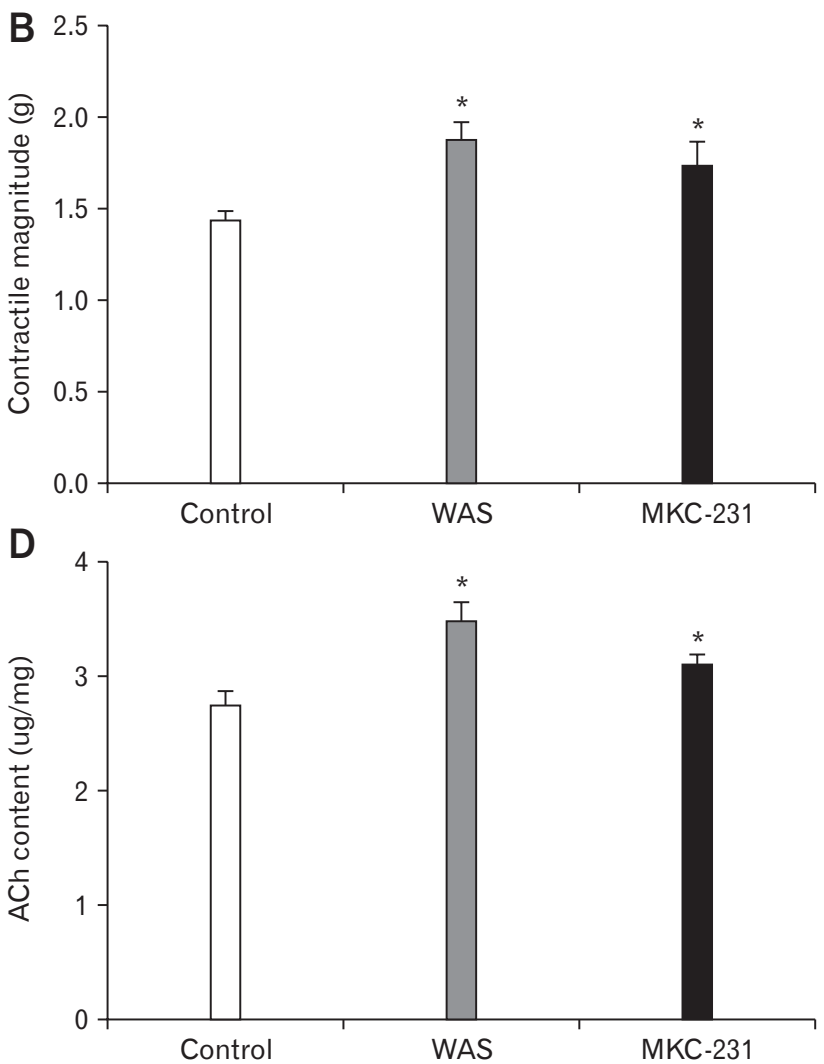

Figure 5. Effect of MKC-231, a choline uptake enhancer, on spontaneous contraction of colonic strips and acetylcholine (ACh) production. (A) A representative image showing spontaneous contractions of colonic strips in vitro. (B, C) The histograms show summary data expressed by the average magnitude and frequency of spontaneous contractions curve. The average amplitude in water avoidance stress (WAS) rats and MKC231-treated rats is significantly higher than that in control rats. Moreover, the frequency in the WAS-exposed rats is markedly increased compared to that in controls. However, the frequency in MKC-231-treated rats is close to that in control rats. (D) ACh content in the colon of WAS-exposed rats and MKC-231-treated rats is considerably increased compared to that in the control rats. ${ }^{*}<0.05$ compared to controls.

to GI disorders, such as inflammatory bowel disease, ${ }^{26}$ diverticular disease, ${ }^{27}$ and antibiotic-induced gut dysmotility. ${ }^{28}$ Findings from recent studies that $\mathrm{CHT} 1$ functions as a major determinant of cholinergic signaling capacity have ignited significant interest in this family of choline transporters. ${ }^{5,6}$ The purpose of this study is to explore the role of CHT1 in intestinal hypermotility induced by chronic stress.

CHT1 is mainly present in the neuronal cytoplasm and presynaptic membrane and is widely distributed in both peripheral and central nervous systems. ${ }^{29}$ CHT1 is cholinergic-specific according to the results of in situ hybridization and immunohistochemical experiments. ${ }^{6}$ In our current study, we detected that CHT1 was expressed strongly in the colon, especially in the mucosa and muscular layers. Furthermore, two different populations of CHT1-IR cells, namely ICC-IM and MP neurons, were present in the muscular layer. Interestingly, we found that ICC-IM exhibited CHT1 im- munoreactivity, suggesting that ICC-IM may belong to the nonneuronal cholinergic system (NNCS) and participate in $\mathrm{ACh}$ release in the gut. Therefore, intramuscular ICC may be involved in the regulation of GI motility in autocrine or paracrine fashion. A previous study has revealed that the intestinal NNCS is mainly present in the mucosa, where this system modulates chloride secretion. ${ }^{30}$ Presently, only one published study has reported the presence of CHT1 immunoreactivity in the human and rat ENS. ${ }^{7}$ However, this article does not provide detailed information of CHT1 distribution in the colon. Our present research reports for the first time the distribution of $\mathrm{CHT} 1$ in the gut, which importantly adds to the currently limited published literature available on this condition.

In the present study, we detected upregulation of $\mathrm{CHT} 1$ in both protein and mRNA levels in the colon after 10 consecutive days of WAS exposure. Further study demonstrated the increased density of CHT1-positive cells in the muscular layer in WAS- 
A

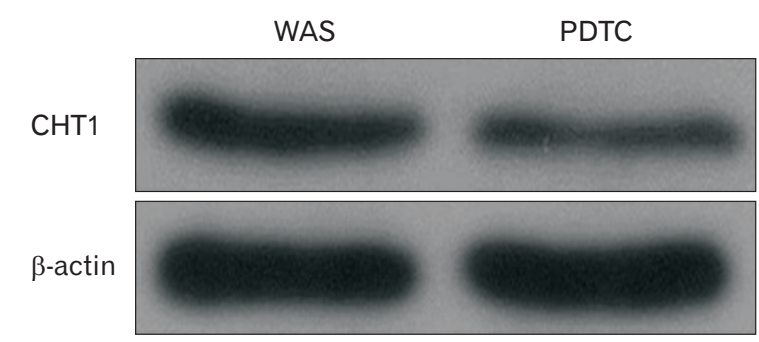

B

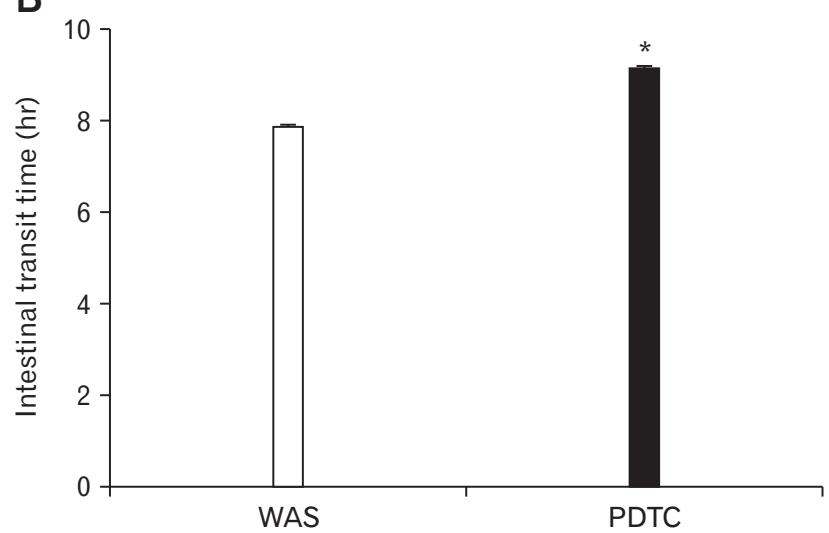

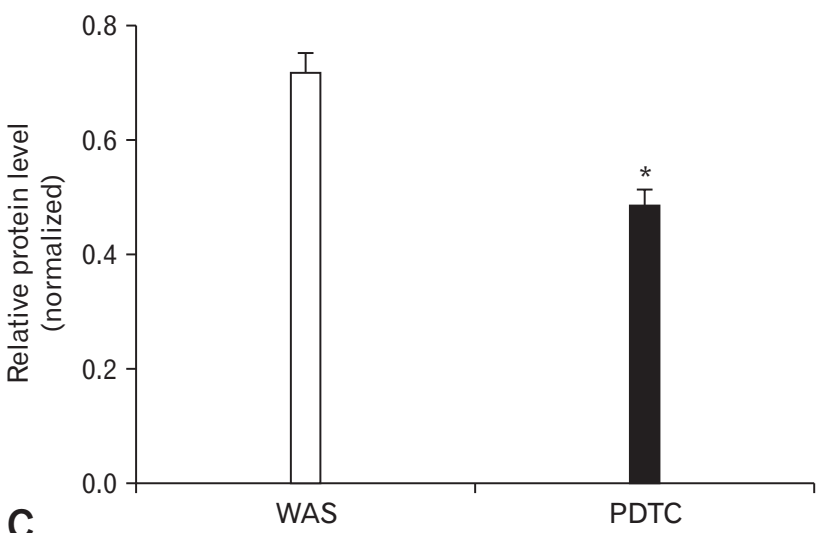

C

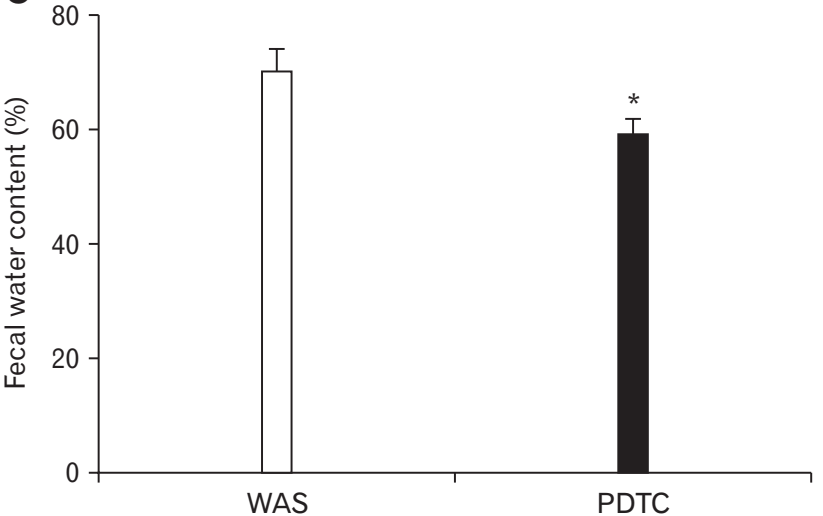

Figure 6. Influence of ammonium pyrrolidine dithiocarbamate (PDTC) on high-affinity choline transporter 1 (CHT1) expression and intestinal motility. (A) Immunoblots (left) and histogram (right) for CHT1 in the colon from water avoidance stress (WAS) and PDTC-treated rats showing that the obtained CHT1 and $\beta$-actin level ratios of the PDTC group are significantly decreased compared to that in WAS rats. (B) The intestinal transit time of WAS rats are increased after treatment with PDTC. (C) The fecal water content of PDTC group is decreased compared with the WAS rats. ${ }^{*} P<0.05$ compared to WAS rats.

exposed rats, accompanied by enhanced ACh release. Our findings indicate that chronic stress-driven changes in gut motility suggest direct modulation of the ICC and ENS, providing important evidence in support of this mechanism in mediating stress-driven changes in brain-gut signaling. A previous study has suggested that reduced proportion of nitrergic enteric neurons in the MP of the distal ileum contributes to the intestinal dysmotility observed in IBS. ${ }^{31}$ However, our findings further demonstrate that intestinal dysmotility in IBS is also related to the hyperfunction of cholinergic neurons in the colonic MP. One potential explanation may be that chronic stress directly regulates the cholinergic system in ICCs and ENS via the brain-gut axis through key alterations in the expression and function of $\mathrm{CHT} 1$. The enhanced non-neuronal ACh release from ICCs coupled with ACh release from the ENS then plays a key role in intestinal hypermotility in WAS-induced IBS via the action of $\mathrm{ACh}$ on muscarinic receptors. ${ }^{32}$

MKC-231, known as a choline uptake enhancer, has been re- ported to take CHT1 as its principal target. ${ }^{33}$ Animal experiments have reported that MKC-231 significantly improves the learning and memory deficits associated with cholinergic hypofunction by affecting the trafficking of CHT1 and increasing the number of transporters working for $\mathrm{HACU}$ at the synaptic membrane. ${ }^{33-35}$ Furthermore, MKC-231 could antagonize phencyclidine-induced behavioural deficits through increases in septal cholinergic neurons. ${ }^{36}$ According to our data, MKC-231 also enhances the normal colonic motility of control rats through upregulation of CHT1 and elevated $\mathrm{ACh}$ production. In turn, we can safely conclude that enhanced CHT1 expression may cause colonic hypermotility induced by chronic stress. Therefore, downregulation of CHT1 or attenuated CHT1 activity may be used to treat disorders involving GI hypermotility. Here, we confirmed that suppression of CHT1 expression via the blockade of $\mathrm{NF}-\kappa \mathrm{B}$ signaling alleviated colonic hypermotility in WAS rats, suggesting that $\mathrm{NF}-\kappa \mathrm{B}$ signaling was involved in the regulation of intestinal motility in stress-induced 
IBS by affecting CHT1 expression. Several lines of evidence have demonstrated that NF- $\mathrm{KB}$ signaling plays an important role in the pathological process of IBS. Firstly, Wang and $\mathrm{Liu}^{37}$ reported that activation of NF- $\mathrm{KB}$ signaling contributed to the occurrence of inflammation-related IBS-diarrhea. In addition, recently, we detected that NF- $\kappa \mathrm{B}$ signaling was also implicated in IBS-related hyperalgesia in an unpublished research, consistent with previous studies reporting that $\mathrm{NF}-\kappa \mathrm{B}$ signaling participated in the pain modulation in inflammatory diseases, ${ }^{38}$ cancer, ${ }^{39}$ and autoimmune diseases, ${ }^{40}$ via the release of inflammatory cytokines such as IL-1 $\beta$, IL-6, and TNF- $\alpha$.

Moreover, our findings indicate that the excitatory effect of CHT1 in GI motility is closely associated with increased ACh release. ACh, an important neurotransmitter in normal GI smooth muscle, mediates contraction via muscarinic 3 receptors on ICC and postsynaptic membrane. ${ }^{41,42}$ Previous studies have suggested that the symptom of diarrhea in IBS patients is relevant to the elevated ACh bioavailability and subsequent increase in GI contractility due to the predominant role of serotonergic system. ${ }^{43}$ Animal studies indicate that chronotropic effects of cholinergic nerve stimulation and ACh release occur via ICC-IM. ${ }^{44}$ In addition, NOdependent neurotransmission can exert inhibitory function on the GI contractility to counteract the effects of the cholinergic system. ${ }^{31}$ All of these facts suggest a complex role for CHT1 in the regulation of GI motility in IBS. More recently, a study demonstrated that $\mathrm{CHT} 1$ also regulates the enzyme activity of enhanced mucosal acetylcholinesterase and influences the release of ACh by affecting the mobilization of vesicle pools, in addition to serving as the choline transporter. ${ }^{45}$ These findings provide a foundation for research into the role of CHT1 in GI motility. However, one limitation of the present study is that choline uptake of CHT1 is not examined. Therefore, further studies focusing on the functional alterations of CHT1 in rats with dysmotility are warranted.

In conclusion, this study demonstrated that $\mathrm{CHT} 1$ may be involved in the generation of colonic hypermotility induced by chronic stress via modulation of ACh production through the NF$\kappa \mathrm{B}$ signal pathway. These findings identify new mechanisms underlying functional colonic hypermotility associated with enhanced stress responsiveness and may pave the way for novel treatments for IBS and related disorders.

Financial support: This work was supported by National Natural Science Foundation of China (NSFC) (No. 81170351 and 81770638).

\section{Conflicts of interest: None.}

Author contributions: Meng-juan Lin conducted the experiments; and Meng-juan Lin and Bao-ping Yu conceived and designed the experiments, contributed toward data analysis, drafting and critically revising the paper, and agree to be accountable for all aspects of the work.

\section{References}

1. Clemens CH, Samsom M, Van Berge Henegouwen GP, Smout AJ. Abnormalities of left colonic motility in ambulant nonconstipated patients with irritable bowel syndrome. Dig Dis Sci 2003;48:74-82.

2. Sanders KM. A case for interstitial cells of Cajal as pacemakers and mediators of neurotransmission in the gastrointestinal tract. Gastroenterology 1996;111:492-515.

3. Oshima T, Miwa H. Epidemiology of functional gastrointestinal disorders in Japan and in the world. J Neurogastroenterol Motil 2015;21:320329 .

4. Medland JE, Pohl CS, Edwards LL, et al. Early life adversity in piglets induces long-term upregulation of the enteric cholinergic nervous system and heightened, sex-specific secretomotor neuron responses. Neurogastroenterol Motil 2016;28:1317-1329.

5. Okuda T, Haga T, Kanai Y, Endou H, Ishihara T, Katsura I. Identification and characterization of the high-affinity choline transporter. Nat Neurosci 2000;3:120-125.

6. Okuda T, Haga T. Functional characterization of the human high-affinity choline transporter. FEBS Lett 2000;484:92-97.

7. Harrington AM, Lee M, Ong SY, et al. Immunoreactivity for highaffinity choline transporter colocalises with VAChT in human enteric nervous system. Cell Tissue Res 2010;341:33-48.

8. Ferguson SM, Bazalakova M, Savchenko V, Tapia JC, Wright J, Blakely RD. Lethal impairment of cholinergic neurotransmission in hemicholinium-3-sensitive choline transporter knockout mice. Proc Natl Acad Sci USA 2004;101:8762-8767.

9. Takashina K, Bessho T, Mori R, Eguchi J, Saito K. MKC-231, a choline uptake enhancer: (2) Effect on synthesis and release of acetylcholine in AF64A-treated rats. J Neural Transm (Vienna) 2008;115:1027-1035.

10. Wang $Z$, Yang $L$, Zheng $H$. Role of app and $A \beta$ in synaptic physiology. Curr Alzheimer Res 2012;9:217-226.

11. Neumann SA, Linder KJ, Muldoon MF, et al. Polymorphic variation in choline transporter gene (CHT1) is associated with early, subclinical measures of carotid atherosclerosis in humans. Int J Cardiovasc Imaging 2012;28:243-250

12. English BA, Appalsamy M, Diedrich A, et al. Tachycardia, reduced vagal capacity, and age-dependent ventricular dysfunction arising from diminished expression of the presynaptic choline transporter. Am J Physiol Heart Circ Physiol 2010;299:H799-H810.

13. Penet MF, Shah T, Bharti S, et al. Metabolic imaging of pancreatic ductal adenocarcinoma detects altered choline metabolism. Clin Cancer Res 2015;21:386-395. 
14. Luo D, Chen L, Yu B. Inhibition of the high affinity choline transporter enhances hyperalgesia in a rat model of chronic pancreatitis. Biochem Biophys Res Commun 2017;488:204-210.

15. Tang D, Qian AH, Song DD, et al. Role of the potassium chloride cotransporter isoform 2-mediated spinal chloride homeostasis in a rat model of visceral hypersensitivity. Am J Physiol Gastrointest Liver Physiol 2015;308:G767-G778.

16. Qi D, Wu S, Zhang Y, Li W. Electroacupuncture analgesia with different frequencies is mediated via different opioid pathways in acute visceral hyperalgesia rats. Life Sci 2016;160:64-71.

17. Gomez-Pinilla PJ, Gibbons SJ, Bardsley MR, et al. Ano1 is a selective marker of interstitial cells of Cajal in the human and mouse gastrointestinal tract. Am J Physiol Gastrointest Liver Physiol 2009;296:G1370G1381.

18. Mach T. The brain-gut axis in irritable bowel syndrome--clinical aspects. Med Sci Monit 2004;10:RA125-RA131.

19. Zhou Q, Verne GN. New insights into visceral hypersensitivity--clinical implications in IBS. Nat Rev Gastroenterol Hepatol 2011;8:349-355.

20. Bhattarai Y, Muniz Pedrogo DA, Kashyap PC. Irritable bowel syndrome: a gut microbiota-related disorder? Am J Physiol Gastrointest Liver Physiol 2017;312:G52-G62.

21. González-Castro AM, Martínez C, Salvo-Romero E, et al. Mucosal pathobiology and molecular signature of epithelial barrier dysfunction in the small intestine in irritable bowel syndrome. J Gastroenterol Hepatol 2017;32:53-63.

22. Hyland NP, Quigley EM, Brint E. Microbiota-host interactions in irritable bowel syndrome: epithelial barrier, immune regulation and braingut interactions. World J Gastroenterol 2014;20:8859-8866.

23. Rojas-Macías V, Rodríguez-Fandiño O, Jiménez-Ponce F, et al. [External validity of a relevant model for irritable bowel syndrome (IBS) using chronic stress by water avoidance in wistar rats.] Rev Gastroenterol Mex 2010;75:421-426. [Spanish]

24. Lü H, Qian JM, Jin GL, Zhou DF, Shen YC. [The establishment of an animal model of gut-brain interaction in irritable bowel syndrome for the evaluation of visceral sensation, motility and psychological behavior.] Zhonghua Nei Ke Za Zhi 2009;48:1035-1039. [Chinese]

25. Harrington AM, Hutson JM, Southwell BR. Cholinergic neurotransmission and muscarinic receptors in the enteric nervous system. Prog Histochem Cytochem 2010;44:173-202.

26. Murray K, Reardon C. The cholinergic anti-inflammatory pathway revisited. Neurogastroenterol Motil 2018;30.

27. Golder M, Burleigh DE, Belai A, et al. Smooth muscle cholinergic denervation hypersensitivity in diverticular disease. Lancet 2003;361:19451951.

28. Caputi V, Marsilio I, Filpa V, et al. Antibiotic-induced dysbiosis of the microbiota impairs gut neuromuscular function in juvenile mice. $\mathrm{Br} \mathrm{J}$ Pharmacol 2017;174:3623-3639.

29. Misawa H, Nakata K, Matsuura J, Nagao M, Okuda T, Haga T. Distribution of the high-affinity choline transporter in the central nervous system of the rat. Neuroscience 2001;105:87-98.

30. Yajima T, Inoue R, Matsumoto M, Yajima M. Non-neuronal release of $\mathrm{ACh}$ plays a key role in secretory response to luminal propionate in rat colon. J Physiol 2011;589(pt 4):953-962

31. Li S, Fei G, Fang X, et al. Changes in enteric neurons of small intestine in a rat model of irritable bowel syndrome with diarrhea. J Neurogastroenterol Motil 2016;22:310-320.

32. Zhu MH, Sung IK, Zheng $\mathrm{H}$, et al. Muscarinic activation of $\mathrm{Ca}^{2+}$ activated $\mathrm{Cl}^{-}$current in interstitial cells of Cajal. J Physiol 2011;589(Pt 18):4565-4582.

33. Bessho T, Takashina $\mathrm{K}$, Tabata $\mathrm{R}$, et al. Effect of the novel high affinity choline uptake enhancer 2 - (2-oxopyrrolidin-1-yl)-N-(2, 3-dimethyl-5, 6, 7, 8-tetrahydrofuro[2, 3-b] quinolin-4-yl) acetoamide on deficits of water maze learning in rats. Arzneimittelforschung 1996;46:369-373.

34. Murai S, Saito H, Abe E, Masuda Y, Odashima J, Itoh T. MKC-231, a choline uptake enhancer, lameliorates working memory deficits and decreased hippocampal acetylcholine induced by ethylcholine aziridinium ion in mice. J Neural Transm Gen Sect 1994;98:1-13.

35. Takashina K, Bessho T, Mori R, Kawai K, Eguchi J, Saito K. MKC231, a choline uptake enhancer: (3) Mode of action of MKC-231 in the enhancement of high-affinity choline uptake. J Neural Transm (Vienna) 2008;115:1037-1046.

36. Shirayama Y, Yamamoto A, Nishimura T, Katayama S, Kawahara R. Subsequent exposure to the choline uptake enhancer MKC-231 antagonizes phencyclidine-induced behavioral deficits and reduction in septal cholinergic neurons in rats. Eur Neuropsychopharmacol 2007;17:616626.

37. Wang XM, Liu YL. [An investigation of signal transduction pathway of colonic mucosal toll-like receptor 4 in patients with diarrhea-irritable bowel syndrome.] Zhonghua Nei Ke Za Zhi 2010;49:491-494. [Chinese]

38. Khalid S, Ullah MZ, Khan AU, et al. Antihyperalgesic properties of honokiol in inflammatory pain models by targeting of NF- $\mathrm{B}$ and $\mathrm{Nrf} 2$ signaling. Front Pharmacol 2018;9:140.

39. Jian-Min T, Jun L, Sheng-Yang C, et al. Tashinone II A-sulfoacid-natrum elevates the pain threshold through inhibiting nuclear factor kappa B pathway in neuropathic cancer pain. Indian J Cancer 2015;52(suppl 3):E179-E181.

40. Xia ZB, Yuan YJ, Zhang QH, Li H, Dai JL, Min JK. Salvianolic acid $\mathrm{B}$ suppresses inflammatory mediator levels by downregulating $\mathrm{NF}-\mathrm{KB}$ in a rat model of rheumatoid arthritis. Med Sci Monit 2018;24:2524-2532.

41. Porter AJ, Wattchow DA, Brookes SJ, Costa M. Cholinergic and nitrergic interneurones in the myenteric plexus of the human colon. Gut 2002;51:70-75.

42. Burleigh DE. $N^{g}$-nitro-L-arginine reduces nonadrenergic, noncholinergic relaxations of human gut. Gastroenterology 1992;102:679-683.

43. Crowell MD. The role of serotonin in the pathophysiology of irritable bowel syndrome. Am J Manag Care 2001;7(8 suppl):S252-S260.

44. Forrest AS, Ordög T, Sanders KM. Neural regulation of slow-wave frequency in the murine gastric antrum. Am J Physiol Gastrointest Liver Physiol 2006;290:G486-G495.

45. Iwamoto H, Calcutt MW, Blakely RD. Differential impact of genetically modulated choline transporter expression on the release of endogenous versus newly synthesized acetylcholine. Neurochem Int 2016;98:138145 